\title{
PLANIFICACIÓN AMBIENTAL COMO ESTRATEGIA PARA LA CONSERVACIÓN DE UNA FUENTE DE ABASTECIMIENTO
}

\section{Environmental planning as a strategy for the preservation of a supplying source}

\author{
LILIANA RECAMÁN ${ }^{1}$ \\ 1Universidad del Cauca
}

E-mail: lilianarecaman@yahoo.es

Recibido: 27 de Julio de 2011

Aceptado: 18 de Enero de 2012

\section{Resumen}

La cuenca del río Las Piedras es la principal fuente de abastecimiento de agua para la ciudad de Popayán, capital del departamento del Cauca. Este ecosistema estratégico beneficia aproximadamente a 230.000 habitantes y nueve acueductos veredales con 650 familias. La Planificación ambiental ha sido la principal estrategia para la conservación, en la que ha jugado un factor primordial la participación comunitaria y su articulación a procesos concertados con las instituciones, contribuyendo al desarrollo de sus alternativas productivas, mejorando su calidad de vida y las condiciones ambientales del sector, permitiendo avanzar en la gestión social, cultural y biofísica, especialmente en lo que concierne a una mayor estabilidad del recurso hídrico.

Palabras claves: Comunidad, Concertación, Conservación, Cuenca, Gestión, Ordenamiento, Planificación, Regulación hídrica, Territorio.

\section{Abstract}

The basin of the river Las Piedras is the main source of supply of water for the city of Popayán. This strategic ecosystem benefits approximately 230.000 inhabitants and nine local aqueducts with 650 families. The environmental Planning has been the main strategy for the conservation, in which the community participation and its articulation to processes concerted with the institutions has being a primordial factor, contributing to the development of its productive alternatives, improving its quality of life and the environmental conditions of the sector, allowing to advance in the social, cultural and biophysical administration, especially in what concerns a bigger stability of the water resource.

Keywords: Community, Agreement, Conservation, Basin, Administration, Planning, Hydric regulation, Territory. 


\section{INTRODUCCIÓN}

A partir de los resultados obtenidos durante el proceso de manejo ambiental que se ha realizado por espacio de 20 años en la cuenca del río las Piedras, ubicada en el municipio de Popayán, Departamento del Cauca, se resalta como el proceso de planificación ambiental ha contribuido a la recuperación de este ecosistema y a la dinámica de los cambios internos y externos. Por tal razón es necesaria una gestión continua en los componentes sociales, culturales y biofísicos, especialmente en una mayor estabilidad del recurso hídrico.

Con el equipo técnico de la Fundación Pro Cuenca Río Las Piedras, la División Ambiental de la Empresa de Acueducto y Alcantarillado de Popayán S.A E.S.P, y el Programa Conjunto de las Naciones Unidas, se realizó una sistematización de la experiencia del trabajo realizado en esta zona, el cual forma parte de la socialización realizada con los grupos comunitarios de la cuenca y se presenta en el contexto del Doctorado en Ciencias Ambientales de la Universidad del Cauca, la Universidad del Valle y la Universidad Tecnológica de Pereira, como parte del programa académico en el módulo de producción más limpia, en el que se resalta la planificación ambiental, los sistemas agrarios sostenibles, y la huella hídrica entre otros tópicos. Este artículo tiene como objetivo analizar; como la planificación ambiental participativa incide en el reordenamiento del uso del suelo, posibilitando a los actores sociales que intervienen el desarrollo de alternativas productivas y de conservación.

Para poder entender las particularidades de esta cuenca, es necesario conocer lo que hay detrás de su historia. Esta región forma parte integral del territorio de comunidades campesinas de Asocampo y Asoproquintana e indígenas de los cabildos de Puracé y Quintana, las cuales a través de sus relacionamientos y visión del territorio, han desarrollado sus prácticas socioculturales. Los actores sociales de la zona relacionan muchas de las prácticas realizadas en sus procesos productivos y ambientales, con los antecedentes que se produjeron desde la época de la colonia con la creación de las grandes haciendas y el desplazamiento de los territorios indígenas y colonos campesinos, en los que se fueron cambiando los usos del suelo, por la actividad ganadera marcando una tendencia de manejo en la cuenca, generando con el tiempo fuertes impactos ambientales, especialmente en la regulación hídrica y en la lucha posterior por la tenencia de la tierra por parte del movimiento indígena, agudizando una crisis entre las comunidades campesinas y propietarios de las grandes fincas.

Dado lo anterior, el conflicto, ambiental, social, político y económico, limitó la oferta de agua y la intervención institucional para su manejo. Este aspecto motiva a que se cree en 1990 la Fundación Pro Cuenca río Las Piedras, con alianzas institucionales para proteger y conservar la cuenca, poniendo en marcha el primer plan de manejo, en los que se dieron los pasos iniciales orientados al desarrollo de programas del orden social, ambiental, productivo y de infraestructura. Para esta época, cuando se formula el primer plan de manejo, se reporta que el $72 \%$ del uso de la tierra estaba en pastizales y el $19 \%$ en bosque, igualmente los caudales registraban mínimos de $500 \mathrm{Lt} / \mathrm{seg}$ con mayor frecuencia, limitando la oferta de agua para los acueductos veredales y el acueducto de la capital caucana, el conflicto social iba en aumento, con un $40 \%$ del territorio.

Durante los años siguientes, La Fundación Pro Cuenca río las Piedras en acuerdos interinstitucionales con la Alcaldía Municipal de Popayán, la Empresa de Acueducto y Alcantarillado de Popayán S.A E.S.P , La Corporación Autónoma Regional del Cauca C.R.C, Parques Nacionales, La Fundación Universitaria de Popayán, la Universidad del Cauca, Federación Nacional de Cafeteros, Fondo para la Acción Ambiental y la Niñez, la Piscifactoría el Diviso, Acción Social de la Presidencia de la República, entre otros, desarrollaron trabajos de planificación ambiental, tendientes a disminuir los conflictos de uso del suelo, aumento de coberturas de protección, fortalecimiento social con énfasis en organización y participación comunitaria, educación ambiental, capacitación técnica, alternativas productivas mejorando la seguridad alimentaria, el establecimiento de sistemas agroforestales y silvopastoriles, saneamiento básico e investigación. Con estos trabajos se logró paulatinamente un incremento en la participación de sus actores sociales replicando buenas prácticas para el manejo ambiental. Adicionalmente a estos trabajos, las comunidades indígenas a través del Consejo Regional Indígena, CRIC, han desarrollado proyectos con enfoques similares contribuyendo a mejorar las condiciones en parte de sus territorios.

Actualmente en la zona se desarrolla un componente de investigación a través del convenio MACACEA ${ }^{1}$ y el proyecto Integración de Ecosistemas y Adaptación al Cambio Climático en el Macizo Colombiano por parte del Programa Conjunto de Naciones Unidas, con el objetivo de disminuir la sensibilidad al cambio climático y mejora de las medidas de adaptación para enfrentar el fenómeno. Es importante resaltar que si bien se tienen resultados en una mayor regulación ambiental, la dinámica de cambio generada por la variabilidad climática y la no reglamentación del recurso hídrico, exigen procesos continuos en la gestión social, cultural, biofísica y empresarial del recurso hídrico para una cuenca estratégica como esta.

1 MACACEA (Monitoreo ambiental para cambio climático en ecosistemas andinos) Convenio desarrollado por la Universidad del Cauca, el Ministerio de Agricultura y la Empresa de Acueducto y Alcantarillado de Popayán S.A E.S.P 


\section{DESCRIPCIÓN}

\section{La planificación ambiental una buena práctica de adaptación}

Las intervenciones productivas en los predios de la cuenca del río Las Piedras, se realizaban de forma intuitiva, sin planificación, lo cual no permitía evaluaciones sobre los avances o retrocesos en las actividades; de igual manera, otros aspectos como la destinación de áreas para conservación y protección de los recursos se realizaba esporádicamente y al azar. Las labores de sensibilización y capacitación para la planificación, implicaron una revisión del pasado, presente y futuro de la cuenca y de sus habitantes, el relacionamiento e interdependencia entre el estado de conservación y mejoramiento de los recursos, la producción y el conocimiento (Figura 1).

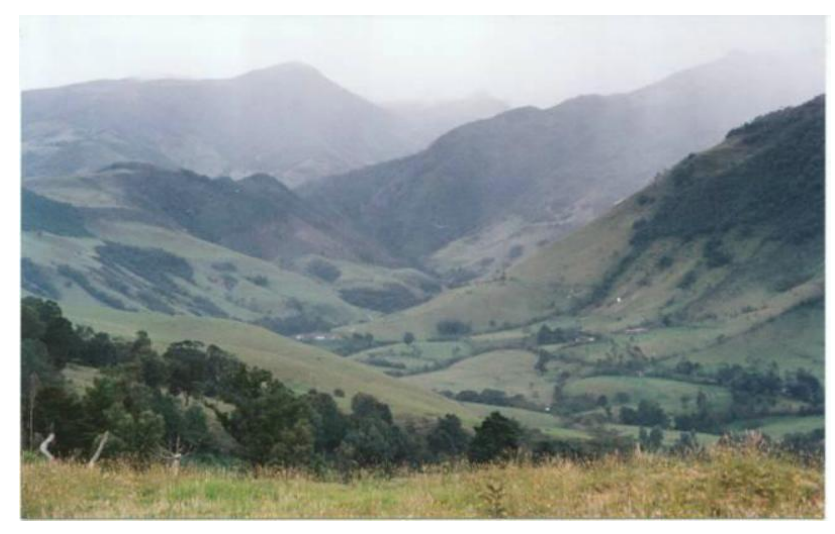

Figura 1. Panorámica cuenca río las Piedras

Campesinos e indígenas consideran que planificar los predios significa conocer puntos de partida, intervenir con conocimiento en los predios y en la cuenca, relacionar la calidad de vida y el mejoramiento de los ingresos, mejorando los recursos naturales.

La variabilidad climática ha generado incertidumbres y modificaciones en las costumbres productivas, la cultura de conservación predial y en la cuenca, factores que pueden ser incorporados en la planificación del futuro de los predios y los recursos.

“La planificación predial es una estrategia que se basa en conocer el estado y relación de todos los componentes de una finca o parcela, sus fortalezas y debilidades, para orientar sus posibilidades de desarrollo, definir las acciones a emprender y el orden de implementación para cada caso familiar. El mejorar la calidad de vida de las familias de la cuenca depende de optimizar los componentes del predio, es decir, sus áreas productivas, el estado de sus bosques, aguas, suelos, infraestructura, la organización familiar y comunitaria, nivel de Capacitación y comunicación entre sus habitantes."[FPRP1].
Para llevar a cabo la planificación predial se adelantaron los siguientes pasos:

- Organización y participación comunitaria para la planificación

-Visita y reconocimiento de los predios o parcelas en compañía del grupo familiar para recolección de información del predio y elaboración del mapa del uso actual.

-Análisis de la información entre el equipo técnico de la Fundación y el grupo familiar para elaborar la propuesta de planificación y priorización de acciones.

-Concertación de las acciones a realizar, teniendo en cuenta aspectos sociales, ambientales y económicos del predio o parcela, basado en las necesidades de la familia y los impactos ambientales a equilibrar e igualmente los lineamientos del Plan de Manejo de la cuenca.

-Elaboración del plan de trabajo

-Socialización del sistema de planificación tanto al grupo familiar como a los integrantes de la organización social vinculada al trabajo.

-Implementación de los sistemas.

-Concertación del plan de seguimiento y evaluación.

-Visitas de acompañamiento y seguimiento.

En este sentido se considera pertinente ilustrar a cerca de las buenas prácticas desarrolladas en la planificación entre las que se destacan: fortalecimiento social e institucional, la conservación y recuperación ambiental de zonas de regulación hídrica y la diversificación de los sistemas productivos con técnicas de producción más limpia, buscando la futura incorporación a mercados diferenciados incrementando la competitividad y mejorando la calidad de vida.

\section{Fortalecimiento social e institucional.}

La recuperación de las condiciones ambientales de la cuenca empieza por el mejoramiento de la calidad de vida de sus habitantes que dependen en gran parte del estado y manejo de los recursos naturales de las reservas o parcelas. La familia es el eje articulador en la toma de decisiones sobre la reserva o parcela, en la que se le da prioridad a los aspectos de organización y fortalecimiento comunitario con equidad de género, la seguridad alimentaria, costumbres y tradiciones, entre ellas las huertas familiares, la producción de abonos orgánicos, minimizando los problemas de contaminación por uso de agroquímicos, la recuperación de la cocina tradicional en la preparación de alimentos propios de la región. A este proceso se vincularon en un mayor porcentaje las familias campesinas y en la medida en que los problemas 
de tenencia de tierra se han ido superando lo han hecho familias indígenas. Este aspecto es el pilar de cualquier proceso de planificación y en la medida en que los mecanismos participativos se fortalezcan entre los actores comunitarios los resultados de sostenibilidad son mayores.

Durante el proceso de fortalecimiento social e institucional, se resaltan: la conformación y consolidación de red de reservas de la sociedad civil, agrupando 64 predios campesinos de Asocampo representando un 20\% del área, la firma del pacto de convivencia entre los actores sociales con el fin de respetar las diferencias y particularidades de cada grupo y establecer acuerdos para una solución concertada del conflicto. Esto dio paso a formular el segundo plan de manejo de acuerdo al decreto 1729 de 2002, con ajustes metodológicos acordados con las comunidades; para esta etapa se contó con un $80 \%$ de participación a diferencia del $10 \%$ de la etapa anterior; el $60 \%$ aproximadamente de las mujeres hoy participan de los procesos y de la toma de decisiones, anteriormente se estima era de un $25 \%$; de las 650 familias con sistemas productivos sostenibles se encuentran en formación un $60 \%$, utilizando actividades de producción limpia ; las soluciones concertadas a la tenencia de la tierra pasaron de un $70 \%$ a un $25 \%$.de las 6626 h.a; los cuatro actores sociales cuentan con un mayor nivel de gestión local, regional, nacional e internacional (Figura 2).

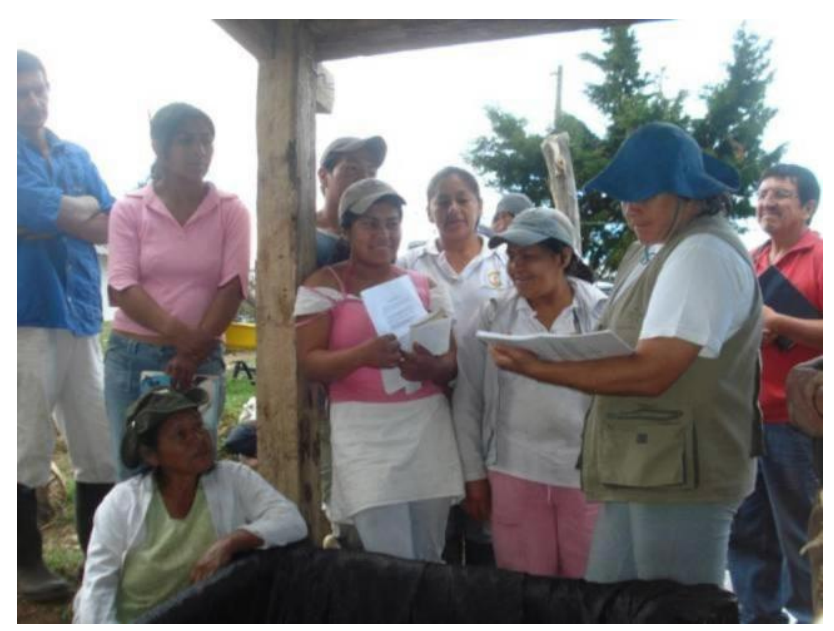

Figura 2. Escuela de campo Cabildo de Quintana en capacitación sobre el establecimiento de biofábricas. Contrato Organización de las Naciones Unidas para la Agricultura y Alimentación FAO-Fundación Pro Cuenca río las Piedras 2009.

Dada la importancia que representó la firma del Pacto de Paz y Convivencia, firmado el 15 de octubre de 2001, el cual es ejemplo a nivel de la región y del país; a continuación se describen los principales aprendizajes: reconocimiento de que existen diferentes intereses sobre el territorio de la cuenca, bajo la visión de interculturalidad, generando respeto por la diferencia y por los derechos de la madre tierra a través del dialogo, el saber escuchar, la tolerancia y el respeto, se han fijado objetivos comunes entorno al desarrollo de la región de manera armónica desde una visión propia. La resolución de conflictos ha permitido avances en la identificación, construcción de pactos entre comunidades y con la naturaleza, para la gestión de propuestas que garanticen el bienestar de las comunidades y una vida digna en el territorio (Figuras 3, 4).

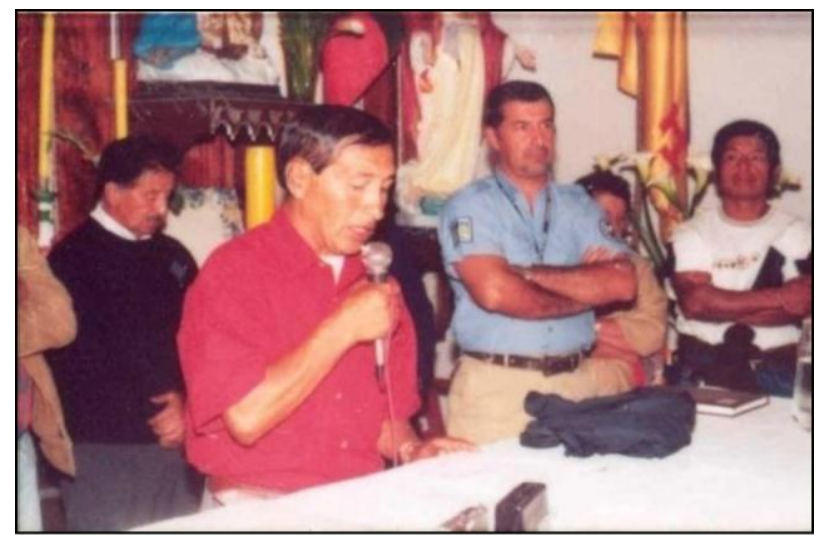

Figura 3. Lectura del Pacto de Paz y Convivencia, representante cabildo de Puracé, octubre 15 de 2001.

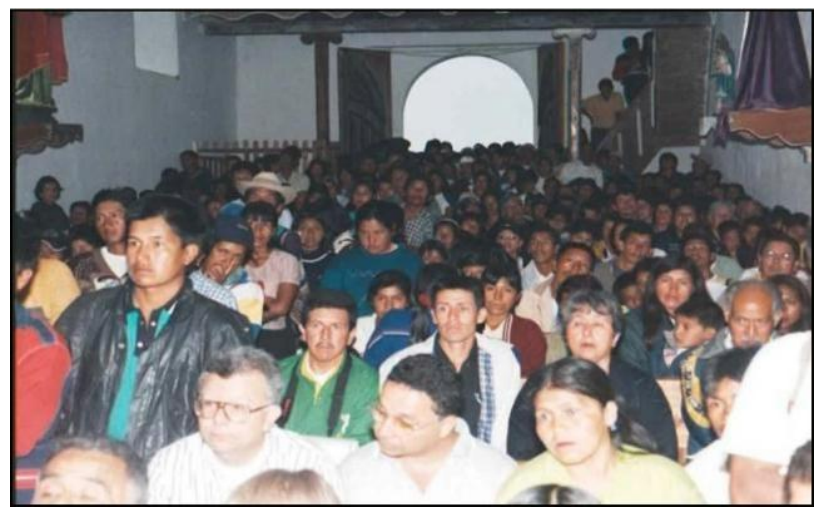

Figura 4. Comunidades campesinas e indígenas de la cuenca Piedras, actores del Pacto de Paz y Convivencia, Octubre de 2001.

\section{Conservación y recuperación ambiental de las zonas de regulación hídrica.}

De acuerdo a lo priorizado en el sistema de planificación, se establecieron cuáles eran los ecosistemas a proteger, entre ellos: nacimientos de agua, humedales, bosques de galería, riberas de cauce expuestas a la ganadería, zonas de páramo, relictos de bosque asociados a la ganadería, áreas de alta pendiente con problemas de erosión. Posteriormente, se realizó una concertación sobre las áreas prioritarias a trabajar, en un esquema de canje ecológico, de esta manera, se fueron liberando áreas de protección a cambio de la implementación de sistemas productivos y de soluciones de 
agua de tipo múltiple para abastecimiento de agua de la vivienda y de la producción agropecuaria, aclarando que en muchos casos se optimizó el sistema ya existente y donde fue necesario se construyó. Gran parte de los ecosistemas de protección se aislaron para dar paso a la regeneración natural y donde fue necesario se desarrolló repoblamiento forestal con especies nativas, propiciando la restauración ecológica y la conectividad de sistemas (Figura 5).

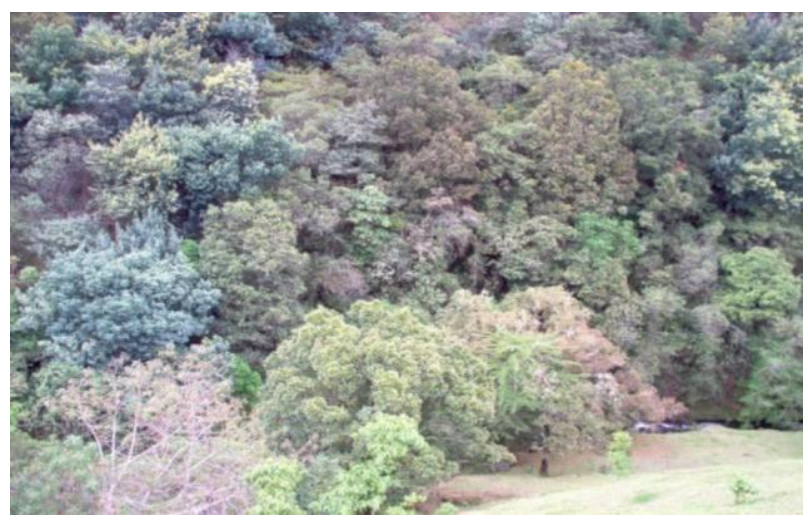

Figura 5. Reserva el Retiro, vereda Santa Teresa, municipio de Totoró, características del proceso de recuperación ambiental de la cuenca.

Como parte integral de la planificación de las zonas de protección se integraron los sistemas agrarios sostenibles, con actividades de producción más limpia que han contribuido al control de la contaminación tanto de los suelos como del agua. El logro de conservar las zonas existentes, realizando aislamientos para propiciar la regeneración natural, restauración con especies propias y recuperación de zonas intervenidas, han mostrado sus resultados en la regulación hídrica, especialmente en zonas de nacimientos de agua con el incremento del caudal de muchas de las micro cuencas de la red hídrica que están asociadas al abastecimiento de los acueductos veredales y del Acueducto de Popayán en la que no se han registrado durante los últimos años caudales que afecten la demanda del recurso. Se destaca a su vez una diferencia en las zonas donde por la tenencia de tierra no se pudieron adelantar trabajos y muestran como contraste, la disminución de caudales en pequeños sistemas de abastecimiento con problemas durante las épocas secas.

Los cambios en los usos del suelo de este proceso a nivel de conservación y de desarrollo agropecuario, está muy asociado a los sistemas de planificación y se describen a continuación.

\section{Sistemas productivos integrados a la producción más limpia}

“La Producción más Limpia es definida por el Programa de las Naciones Unidas para el Desarrollo Industrial ONUDI), como la aplicación continua de una estrategia ambiental preventiva integrada a procesos, productos y servicios que procura aumentar la eficiencia y reducir los riesgos a los seres humanos y al ambiente. Por esto es que en los Programas de PML se implementan de forma consistente prácticas y tecnologías que contribuyen a la adecuación ambiental de las empresas, generándole a la vez beneficios económicos" (Oestreich 2005).

El logro de cumplir tan importante objetivo, es todo un proceso que para el caso de la cuenca, ha tenido avances en el desarrollo de sus prácticas productivas, mitigado los impactos por el desarrollo de la ganadería. Uno de los aspectos que ha mejorado en la zona es la seguridad alimentaria ya que la tradición de explotación ganadera llevó a que muchas familias se dedicaran exclusivamente a ella y dejaran de producir sus propios alimentos, perdiendo su autonomía alimentaria y estableciendo una dependencia en la compra de los productos de su canasta familiar a las plazas de mercado de la ciudad de Popayán. Para el caso de muchas familias su bajo poder adquisitivo por no tener fuentes de empleo en la zona conllevó a bajos niveles de nutrición, esto fue identificado durante el proceso de formulación del Plan de Ordenación y Manejo de la Cuenca.

A continuación se muestra el cambio del uso del suelo en 20 años, periodo comprendido entre 1989 al 2009: "en 1989 el $28 \%$ del área se encontraba en bosques fragmentados por la actividad ganadera, y la extracción de material vegetal para producir carbón y venta de madera. El $2 \%$ de la cobertura en paramos y el $70 \%$ de la cobertura en zonas de pastizales" (Piedras 1990) (Figura 6).

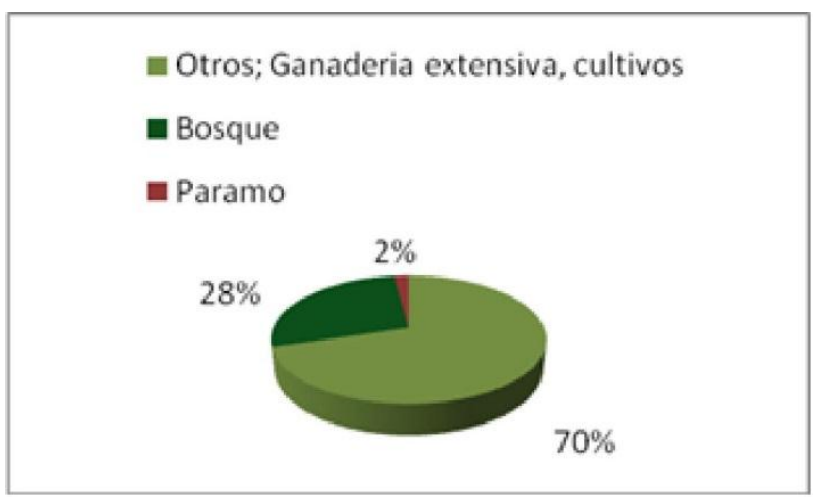

Figura 6. Usos del suelo cuenca Piedras año 1990

“Para el período entre 1990 al 2001, se presenta un incremento del $1 \%$ en el área de bosque pasando de un $28 \%$ a un $29 \%$, lo anterior se logró con los trabajos de restauración ecológica, protección y mantenimiento. En la cobertura de páramos se pasó de un $2 \%$ a un $3 \%$, aumentando en $1 \%$ el área. La cobertura en el ítem otros, que estaba representada en pastizales, con acciones en sistemas integrados de producción como la división de potreros, implementación de sistemas silvopastoriles y 
sistemas silvoagrícolas, se pasó de un $70 \%$ a un $67 \%$, iniciando el manejo de la ganadería extensiva, disminuyendo levemente el conflicto por el uso del suelo, indicando un camino de modificación en la tecnología ganadera existente desde hace cientos de años" (Piedras 2009) (Figura 7).

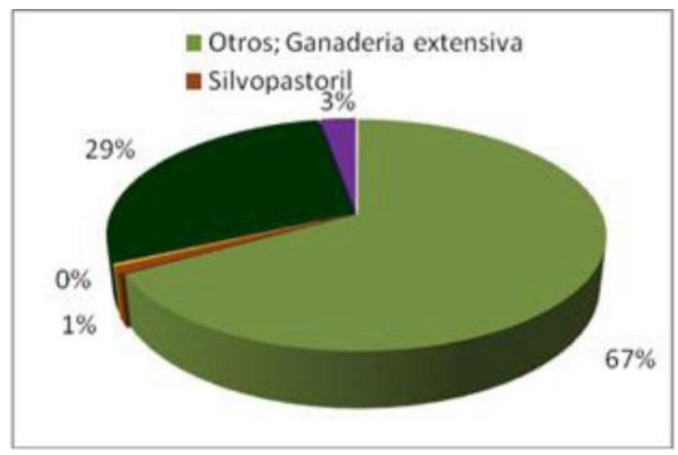

Figura 7. Usos del suelo cuenca Piedras año 2001.

“Para el período entre el 2001 al 2009, el área de bosques se incrementa de un $29 \%$ a un $40 \%$, con un incremento del $11 \%$, lo cual se logra por medio de trabajos en restauración ecológica, protección y mantenimiento de las áreas intervenidas en años anteriores y el fortalecimiento de los miembros de la red de reservas de la sociedad civil. En páramos, se mantiene en $3 \%$ el área de cobertura. Las zonas de Pastizales pasan a un $54 \%$ (ítem otros), se continúan los trabajos de división de potreros con cerca eléctrica y/o solar, bancos de forraje, bebederos ecológicos, implementación de sistemas silvopastoriles, sistemas 2silvoagrícolas, recuperación de suelos, mejorando las condiciones de manejo de la ganadería, con la presencia de un $2 \%$ en sistema silvopastoriles y un $1 \%$ en sistema silvoagrícolas, lo cual reduce el conflicto del suelo en un 3\% más" (Piedras, 2009) (Figura 8).

Es importante resaltar que el generar estas alternativas productivas ha permitido que el uso del suelo siga cambiando y disminuyan los conflictos de uso del suelo y los problemas de contaminación.

Durante este periodo se firma el pacto de paz y convivencia y se logran acuerdos significativos de negociación para el conflicto de tierras. La planificación ambiental identificó como optimizar las buenas prácticas con enfoques de producción más limpia, fortaleciendo la huerta familiar, capacitación de preparación de abonos orgánicos e implementación de biofábricas, preparación de alimentos rescatando recetas tradicionales, control de erosión, el establecimiento de sistemas silvopastoriles y silvoagrícolas, conservación de áreas de protección con conectividad a corredores de conservación (Figuras 9 y 10). Lo anterior ha permitido minimizar problemas de contaminación por el uso de agroquímicos y minimizar los conflictos por el mal manejo de los recursos naturales. Estas buenas prácticas con resultados importantes en años anteriores son objeto de réplica a través de escuelas de aprendizaje para una mayor cobertura de familias campesinas e indígenas que hoy participan de la ejecución de Convenios con el PC ${ }^{2}$ (Figura 11).

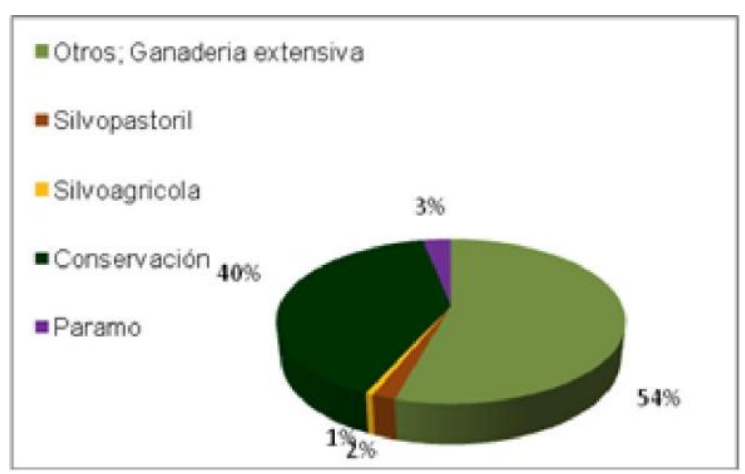

Figura 8. Usos del suelo cuenca Piedras año 2009.

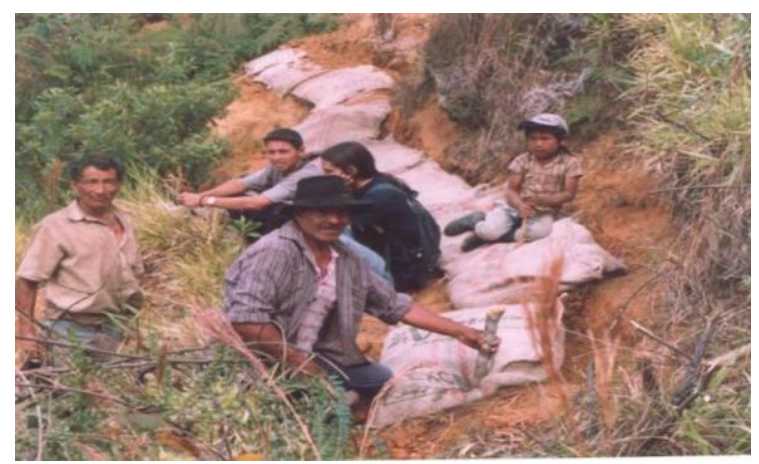

Figura 9. Obras biomecánicas para control de erosión, predio La María, Vereda las Guacas, familia Ortega.

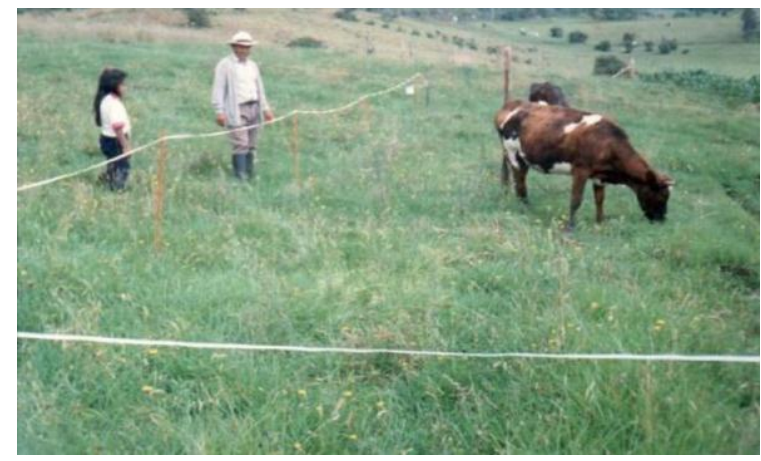

Figura 10. Manejo de praderas con rotación y cerca eléctrica, reserva Santa Marta, familia Escobar, vereda de Quintana

2 PC: Programa Conjunto de Naciones Unidas que ejecuta convenios para establecer medidas de adaptación por los efectos del Cambio Climático con los actores sociales de la cuenca, Alcaldía Municipal de Popayán, La Fundación Procuenca Río Las Piedras y la Empresa de Acueducto y Alcantarillado de Popayán S.A E.S.P 
Frente al proceso anterior vale la pena analizar cómo estas buenas prácticas se convierten en herramientas para avanzar en la producción más limpia y en la competitividad que demanda una mayor gestión para optimizar los procesos productivos para beneficio de las comunidades (Figura 12).

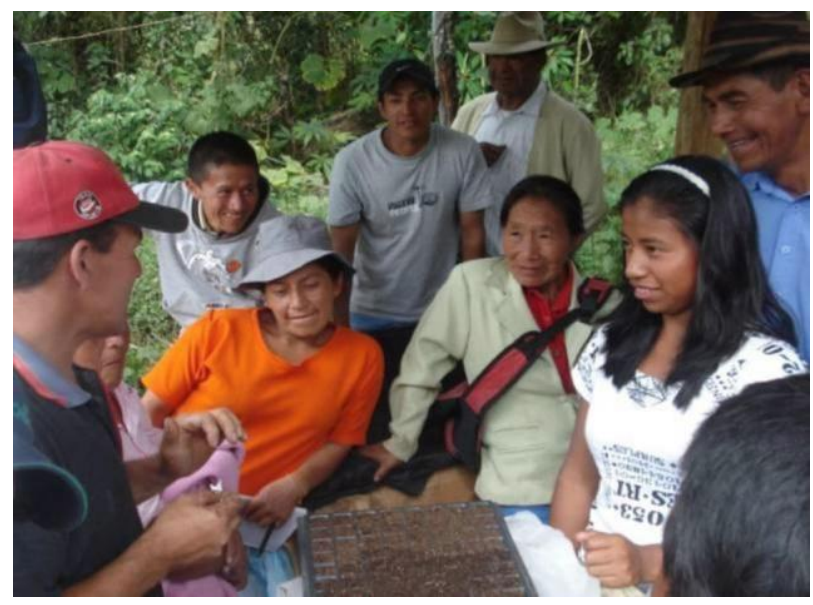

Figura 11. Escuela de Campo de Asocampo en intercambio de conocimientos para el desarrollo de sistemas de seguridad alimentaria.

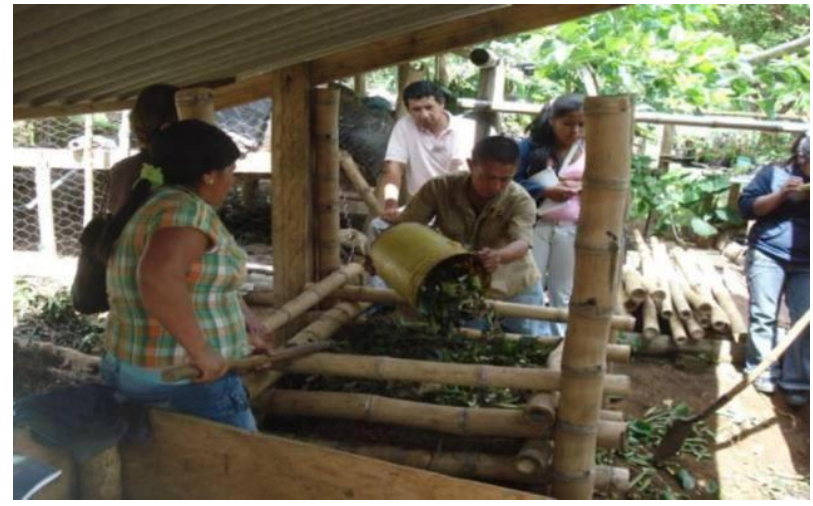

Figura 12. Preparación de abonos orgánicos, reserva Asturias, vereda las Guacas, cuenca río Piedras.

\section{LA DINÁMICA DEL ECOSISTEMA FRENTE A LOS CAMBIOS ACTUALES}

A pesar de tener avances en el manejo de la cuenca del río las Piedras, los sistemas y herramientas para una gestión social, cultural, biofísico del recurso hídrico, debe ser dinámica y fortalecida en la estrategia de planificación ambiental, dado que no son ajenos los cambios por la variabilidad climática, lo que conlleva a que exista una mayor vulnerabilidad ante futuros déficit de agua que no logren responder a las demandas para los usos de desarrollo agropecuario, empresarial, de abastecimiento y de sostenibilidad ecológica.

Durante los últimos años se han evidenciado períodos de lluvias con valores de precipitación por encima de lo normal, lo que se atribuye a la variabilidad climática. La afectación inminente ante los cambios generados por esta situación, se ha manifestado con impactos sobre los diferentes componentes biofísicos de la cuenca, entre los más significativos se analizan la saturación de los suelos, desprendimientos masales, caudales máximos con arrastre de empalizadas y afectación de bocatomas de algunos acueductos veredales y a la infraestructura de captación de la empresa de acueducto de la ciudad de Popayán, como lo ocurrido durante el año 2008 (Figura 13).

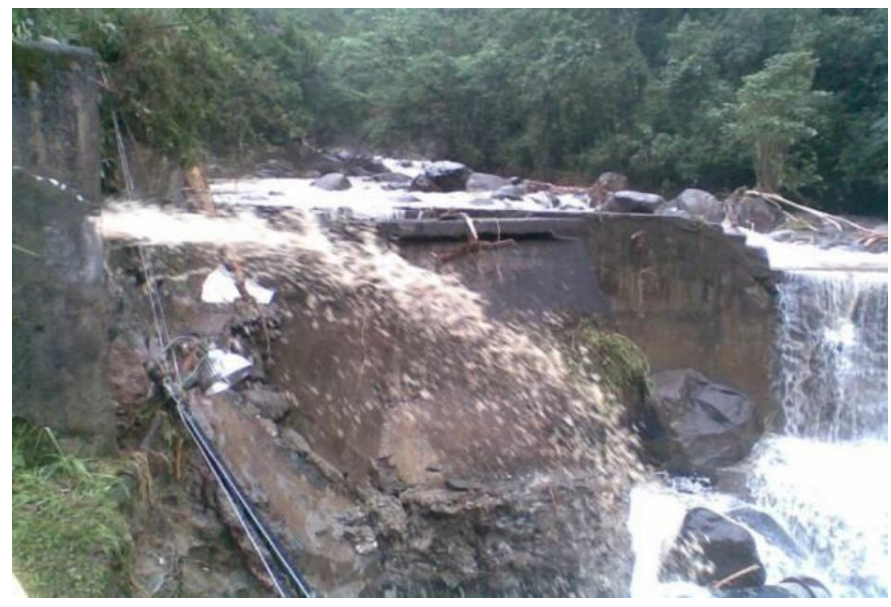

Figura 13. Creciente río Piedras con afectación a la bocatoma del Acueducto de Popayán, año 2008.

Es importante considerar que se tienen dificultades en la reglamentación del recurso hídrico. A pesar de haberse priorizado dentro del Plan de Ordenación y Manejo. Este trabajo no se pudo realizar debido al rechazo que generó por parte de las comunidades indígenas. La reglamentación no puede concebirse únicamente frente a las políticas de las instituciones, también debe trabajarse frente a la visión de las comunidades, aclarando, que es muy importante hacerlo, pero concertadamente y seguramente a través de una metodología diferente que permita una gestión integrada y adaptativa.

Una herramienta nueva que se está planteando en las propuestas del manejo integrado del recurso hídrico, es la huella hídrica, este indicador, se define como el total de agua usada para producir los bienes y servicios consumidos por el individuo, por ese grupo de personas o por el país. (Chapagain \& Hoekstra 2004, citado en Rodríguez et al. 2008). Rodríguez et al. (2008) en su documento sobre la 
Introducción a los estudios de Huella Hídrica realizados en Guadiana España, argumenta: "Con la premisa de entender las cuencas hidrográficas como sistemas socio ecológicos, la gestión adaptativa trata de incorporar el principio de la incertidumbre a la gestión, mediante la capacidad de adaptación, esto es incrementando la capacidad de aprender a la vez que se gestiona. Por ello se debe dar un proceso interactivo basado en el control y la aplicación de métodos de comprobación y análisis y la mejora de las prácticas y estrategias en la gestión dentro de la planificación". (Rodríguez et al. 2008).

Los procesos de Gestión Integral del recurso hídrico, propuestos desde una mirada Interdisciplinar permiten tener un intercambio y cooperación entre los actores sociales e institucionales, construyendo de esta manera acuerdos y puesta en marcha de acciones encaminadas a mejorar las situaciones presentes, frente al problema de futuros déficit de agua. La interdisciplina, juega un papel clave en este sentido ya que permite "abrir los marcos interpretativos para realizar análisis desde múltiples ópticas de unas realidades que se vuelven cada vez más complejas" (Alban 2006). Esta realidad de cambio, de variación de un recurso que se deteriora y se agota, se vuelve una realidad compleja que no es posible interpretarla bajo una sola disciplina de las ciencias y mucho menos sin él saber o el hacer o la práctica de las comunidades, esto nos ofrece un nuevo paradigma para resolver los problemas de este tipo.

El lograr incluir este concepto en los análisis de la valoración del recurso a través de la huella hídrica, junto con el dialogo de saberes de las comunidades, permitirá incorporar mejores herramientas metodológicas para la propuesta de una gestión del recurso hídrico, así mismo, permitirá identificar que otras variables del orden sociocultural inciden sobre la toma de decisiones, entre ellas la visión del territorio, y la lectura que esto significa para entender que cualquier propuesta que se realice debe ser objetiva, aceptada, práctica y replicable

Son precisamente estos espacios de reflexión los que inciden en la toma de decisiones al interior de las comunidades y en los que se pueden establecer compromisos. Esto nos acercaría a la construcción de escenarios de análisis, de cuál es nuestro potencial hídrico, su accesibilidad y verdadera disponibilidad en periodos a corto, mediano y largo plazo y como se vería afectado, si la variabilidad climática se intensifica y no se regula el recurso y como esto afectaría sus sistemas productivos. Nos invitaría entonces a aportar nuevos elementos metodológicos que puedan ser replicables en otras cuencas de alta montaña con características similares, teniendo en cuenta los enfoques de uso y manejo del agua, desarrollo científico como aporte a las ciencias, realizando una gestión social, cultural y biofísica del río las Piedras, como fuente de abastecimiento, integrándolas a la estrategia de la planificación ambiental, ya que solo optimizando los usos y las alternativas para la comunidad es viable esperar resultados y medidas de adaptación frente a las dinámicas ambientales y sociales de un ecosistema.

\section{LA GESTIÓN INTEGRADA DEL RECURSO HÍDRICO Y SU INFLUENCIA EN LA DINÁMICA DE LA PLANIFICACIÓN}

La Asociación Mundial para el Agua (Global Water Partnership, GWP $)^{3}$ define a la Gestión Integrada del Recurso Hídrico, GIRH, como un proceso que promueve el desarrollo y gestión coordinada del agua, la tierra y los recursos asociados, para maximizar el resultante bienestar económico y social de manera equitativa sin comprometer la sostenibilidad de ecosistemas vitales. A nivel de cuencas hidrográficas o lacustres y de acuíferos, GIRH puede definirse como un proceso que permite la gestión coordinada del agua, la tierra y los recursos asociados dentro de los límites de una cuenca para optimizar y compartir equitativamente el resultante bienestar socioeconómico sin comprometer la salud de ecosistemas vitales a largo plazo.

Teniendo en cuenta el ámbito mundial, de acuerdo al informe sobre el desarrollo de los recursos hídricos (WWDR) de las Naciones Unidas, consideran que la eficiencia de la gestión de los recursos mundiales de agua dulce por parte de la sociedad debe comprender un contexto institucional y socioeconómico en la que se analice el abastecimiento, la demanda, los usos del agua y los problemas ocasionados por los fenómenos extremos considerando los problemas críticos actuales y las amenazas a los ecosistemas de agua dulce y su gestión. En la Declaración del Milenio de las Naciones Unidas se insta a todos los Estados Miembros a: "Poner fin a la explotación insostenible de los recursos hídricos formulando estrategias de ordenación de esos recursos en los planos regional, nacional y local, que promuevan un acceso equitativo y un abastecimiento adecuado" (WWAP 2001).

Según el Ministerio de Ambiente, Vivienda y Desarrollo Territorial (MAVDT 2010) La gestión integral del recurso hídrico es un concepto basado en la idea de que los diferentes usos del recurso son excluyentes e interdependientes (y) surgió como respuesta a la "crisis del agua" expresada en la presión insostenible sobre el recurso hídrico, debida a la creciente demanda de agua, la contaminación y el crecimiento demográfico. Sin embargo, se ha observado que el núcleo del problema está en la inadecuada gestión y gobernabilidad del recurso. La gestión integral del recurso hídrico busca actuar sobre las causas de esta gestión deficiente como son la ineficiencia, los conflictos crecientes y el uso no coordinado del recurso hídrico",

\footnotetext{
3 (Global Water Partnership, GWP), Es una red internacional cuya visión es la de un mundo donde esté garantizada la seguridad hídrica. Su misión es apoyar el desarrollo y gestión sostenible de los recursoshídricos en todos los niveles. fue creada en 1996 para estimular la Gestión Integrada de los Recursos Hídricos (GIRH), y asegurar el desarrollo y gestión coordinada del agua, la tierra y los recursos asociados, maximizando el bienestar económico y social, sin comprometer la sostenibilidad de sistemas ambientales vitales.
} 
haciendo necesaria la consolidación de la base de conocimientos sobre los recursos hídricos para la toma de decisiones.

Considerando lo anterior, se plantea vincular a los actores comunitarios y a las instituciones, en la toma de decisiones y responsabilidades, para ejercer un mayor control sobre el recurso y su ordenamiento; con esto se puede entonces configurar y orientar políticas al interior de los actores, armonizadas con las condiciones reales del sector, buscando integrar estos aportes a las políticas regionales y nacionales, fortaleciendo las capacidades para mejorar la gestión, lo cual se puede ver reflejado en el Plan de Ordenación y Manejo de Cuencas, como instrumento de planificación y gestión. Un aspecto relevante en este proceso es tener el conocimiento sobre el valor del agua, ya que se convierte en una herramienta clave para la toma de decisiones de su gestión y ordenamiento. La valoración del recurso se puede realizar a través del cálculo de la Huella Hídrica, como indicador, el cual puede ser ajustado a un ecosistema tan particular como la cuenca del río las Piedras.

Las decisiones concertadas frente al ordenamiento del recurso y la complejidad de los problemas relacionados con el agua exigen políticas destinadas a mantener y ampliar la base de conocimientos. Lo antes descrito permite integrar las diferentes variables de los componentes biofísicos, sociales, culturales y empresariales para ajustar una metodología que permita construir la gestión integrada del recurso, garantizando la disponibilidad de agua para una población superior a los 250.000 habitantes en el municipio de Popayán y a los 650 habitantes de la cuenca, integrando la interdisciplina y la interculturalidad y los criterios de complementariedad de las ciencias.

\section{CONCLUSIONES}

-Para lograr objetivos de conservación y sostenibilidad de una cuenca, es necesario trabajar en procesos de planificación ambiental, involucrando sus actores sociales e institucionales en la toma de decisiones y el desarrollo de alternativas productivas a favor de mejorar sus condiciones sociales.

-La planificación ambiental solo es viable si se responde a las necesidades socioculturales de una región.

-Cualquier objetivo institucional para garantizar la oferta ambiental de una región, demanda un relacionamiento acorde con la interculturalidad y particularidades de los diferentes actores sociales.

- La planificación ambiental permite integrar el ordenamiento del uso del suelo con buenas prácticas ambientales aplicando técnicas de producción más limpia que inciden en el control de la contaminación y una mayor regulación hídrica.

- Con el conocimiento de los usos del suelo de una finca o parcela, se pueden optimizar las actividades productivas, posibilitando que sus propietarios se interesen en adelantar procesos de conservación con sostenibilidad en el tiempo.

-Para lograr una gestión integrada del recurso hídrico es fundamental articular la oferta con las necesidades de demanda de agua para las actividades productivas y de desarrollo social, mediante procesos concertados.

- Los cambios de los ecosistemas como una cuenca hidrográfica ameritan procesos de ajustes que sean acordes a la gestión social, cultural y biofísica de una región, permitiendo el desarrollo de medidas de adaptación que logren responder a esta dinámica.

\section{REFERENCIAS}

ALBAN, A. 2006. Tejiendo Textos y Saberes. Editorial Universidad del Cauca ed. 21.

MAVDT, M.D.A. 2010. Vivienda y Desarrollo Territorial, Política Nacional para la Gestión Integral del Recurso Hídrico. Bogotá.

OESTREICH, A. M.K.D.S.Y.V.M.R. 2005, Producción más limpia y competitividad, un camino hacia la excelencia empresarial sustentable., in Revista de antiguos alumnos del IEEM. Uruguay.

PIEDRAS, F.P.R. \& P.C.D.N. UNIDAS. 2009. Sistematización de buenas prácticas de manejo de recursos naturales cuenca río las Piedras. Fundación Procuenca río las Piedras, Programa Conjunto de Naciones Unidas, Popayán. $100 \mathrm{pp}$.

PIEDRAS, F.P.R., A.Y.A.D. POPAYÁN, AND C.A.R.D.V.D. CAUCA. 1990. Plan de Ordenación y Manejo Cuenca río las Piedras., Fundación Procuenca Río las Piedras, Popayán. 250 pp.

RODRIGUEZ, C. GARRIDO, L. \& VARELA, O. 2008. La Huella Hidrológica de la Agricultura Española. Santander: Fundación Marcelino Botin. 7.

WWAP, U. 2001. La seguridad del agua: evaluación preliminar de los avances en materia de políticas desde Río. Programa mundial de evaluación de los recursos hídricos de las Naciones Unidas (WWAP). 7. 